\title{
Optimal Pumping in a Single Chamber Microbial Fuel Cell
}

\author{
A. Araújo, Maria F. Patrício, José L. Santos \\ CMUC, Department of Mathematics, University of Coimbra \\ 3001-454 Coimbra, Portugal \\ Fax number: +351239793069
}

alma@mat.uc.pt,_mfsp@mat.uc.pt, zeluis@mat.uc.pt

\begin{abstract}
In this work we study the numerical behavior of a diffusion equation with a discrete source term and control variables. Our goal is to use optimization techniques in order to obtain a numerical solution that minimizes a given objective function taking into account some restrictions. Some numerical results are presented concerning the application into a model that describes the oxygen concentration in a single chamber microbial fuel cell. These numerical results are used to obtain a continuous approximation with the least-squares method.
\end{abstract}

\section{Keywords}

Diffusion equation, finite differences, method of lines, nonlinear optimization problem, fitted model.

\section{The approach}

Let us consider the equation that governs the diffusion of a given substance with concentration $u$ with a discrete source term in the form

$$
u_{t}=\alpha u_{x x}+\sum_{i=1}^{r} q_{v_{i}}(t) \delta\left(x-y_{i}\right),
$$

$x \in] 0, L[, t \in] 0, T]$, where $\delta$ is the Dirac delta function, $\alpha>0$ its diffusion coefficient and $r$ the number of points where we consider the "injections". For $i \in\{1, \ldots, r\}, q_{v_{i}}(t)$ is a control variable which gives use a measure of the "sparging rate" of the substance in the point $\left.y_{i} \in\right] 0, L[$, in the instance $t$.

We consider the boundary conditions

$$
u(0, t)=c_{0} \text { e } \frac{\partial u}{\partial x}(L, t)=0, \forall t \geq 0,
$$

as well as the initial condition

$$
u(x, 0)=0, \forall x \in] 0, L[.
$$

The control variables are calculated solving an optimization problem. We consider the problem studied in [7], which is de- scribed in the following way:

$$
\min f(Q)
$$

s.a. :

$$
\begin{gathered}
\sum_{i=1}^{r} \int_{0}^{T} q_{v_{i}}(t) d t=K \\
q_{v_{i}}(t) \geq 0, i \in\{1, \ldots, r\}, t \in[0, T]
\end{gathered}
$$

where $f(Q)=C \sum_{i=1}^{r} \int_{0}^{T}\left(u_{b}-u\left(y_{i}, t\right)\right) q_{v_{i}}(t) d t, K$ and $C$ are positive constants, and $u_{b}$ is an upper bound for the concentration.

The numerical solution for this problem is obtained by finite differences and optimization techniques.

\section{Discrete problem}

Let us consider in $[0, L] \times[0, T]$ a grid $\left\{\left(x_{i}, t_{j}\right): i=\right.$ $0, \ldots, n, j=0, \ldots, m\}$ with $x_{0}=0, x_{n}=L, t_{0}=0, t_{m}=$ $T$, with constant step sizes $\Delta x=x_{i}-x_{i-1}, i=1, \ldots, n$ and $\Delta t=t_{j}-t_{j-1}, j=1, \ldots, m$. Let us also consider $\left\{v_{1}, \ldots, v_{r}\right\} \subseteq\{1, \ldots, n-1\}$ the indexes of the nodes of the spatial discretization where we consider the injections, i.e., $x_{v_{i}}=y_{i}, 1 \leq i \leq r$. Using a second-order centered finite difference operator in space and the implicit Euler method in time, with constant step sizes $\Delta x$ in space and $\Delta t$ in time, the solution $u$ of the equation (1) can be approximated by the solution of the following linear systems:

$$
M U^{j}=U^{j-1}+R \overline{c_{0}}+\Delta t Q^{j}
$$

$$
j \in\{1, \ldots, m\},
$$

where $U^{j}=\left(u_{1}^{j}, \ldots, u_{n-1}^{j}\right)^{\top}$, with $u_{i}^{j} \approx u\left(x_{i}, t_{j}\right)$, $Q^{j}=\left(q_{1}^{j}, \ldots, q_{n-1}^{j}\right)^{\top}, \quad 1 \leq j \leq m$, with $q_{i}^{j}=$ $0, \forall i \in\{1, \ldots, n-1\} \backslash\left\{v_{1}, \ldots, v_{r}\right\}, \forall j \in\{0, \ldots, m\}$, $\overline{c_{0}}=\left(c_{0}, 0, \ldots, 0\right)^{\top}, M_{(n-1) \times(n-1)}$ a tri-diagonal matrix with $m_{i, i}=2 R+1, m_{i, i+1}=m_{i+1, i}=-R(1 \leq i \leq n-2)$ and $m_{(n-1),(n-1)}=1+R$, and $R=\alpha \frac{\Delta t}{\Delta x^{2}}$. Note that the 
control variables are $q_{v_{1}}^{j}, \ldots, q_{v_{r}}^{j}$; the other components were introduced in order to write the vectorial equation (7).

If we consider a discretization of (4)-(6), we obtain a discrete approximation for the optimal solution by solving the following optimization problem:

$$
\min f(Q)=C \sum_{j=1}^{m} \sum_{i=1}^{r}\left(u_{b}-u_{v_{i}}^{j-1}\right) q_{v_{i}}^{j}
$$

s.a. :

$$
\begin{array}{r}
\Delta t \sum_{j=1}^{m} \sum_{i=1}^{r} q_{v_{i}}^{j}=K \\
q_{i}^{j} \geq 0, i \in\{1, \ldots, n-1\}, \\
j \in\{1, \ldots, m\}
\end{array}
$$

This strategy could be generalized to other objective functions. Replacing the solution of (7) in (8), we obtain a quadratic programming problem and we may use known numerical algorithms to solve it [5], [6].

In spite of the discrete source term, since we define our grid in order to contain the injection points, we may conclude that this scheme has order $\mathcal{O}\left(\Delta x^{2}, \Delta t\right)$ (see [2], [4]).

\section{Optimal solution}

To obtain an approximation to the solution of (1)-(6), we solve (8)-(10) using two different strategies. In a first case we will consider $Q$ as a time independent function. Then, we have $q_{i}^{j}=$ $q_{i}, \forall i \in\{1, \ldots, n-1\}, \forall j \in\{1, \ldots, m-1\}$ and we want to know where and how many substance must be injected in the system. In a second case, we consider $Q$ as a time dependent function and so we want to know where, when e and how many substance must be injected in the system.

We now describe the optimal solutions for the two different strategies described above.

For the first case - time independent $Q-$ is it possible to solve (8)-(10) analytically. We may prove that the optimal source location is $v_{1}=1$ (see [1]).

For the case where $Q$ is time dependent we cannot obtain an analytical solution to the optimization problem. Nevertheless, our numerical tests (in [1]) show that the best location to feed the system is still the point $x_{1}$ (i.e., $r=1$ and $v_{1}=1$ ). So, we use the quadprog routine of the MatLab Optimization Toolbox ${ }^{\mathrm{TM}}$ to approximate the optimal solution and the result agrees with the previous case: only $Q^{1}$ is non null, in spite of the last components of this vector be positive (i.e., the $K$ units of substance available are injected in a short period of time, near to the final time).

\section{Numerical results}

The problem (1)-(3) may be viewed as a model for the oxygen diffusion in a microbial fuel cell [3]. A microbial fuel cell converts chemical energy, available in a bio-convertible substrate, directly into electricity. To achieve this, bacteria are used as a catalyst to convert substrate into electrons.

Let us consider, as in [1] and [7], $c_{0}=40$ and different values for the other parameters. In both cases we consider the problem (1), with $\alpha=20, L=310$ and $T=50$. We admit that, in (8)-(10), $K=350$ and $u_{b}=50$. In order to compute the numerical solution, we consider the strategies explained before.
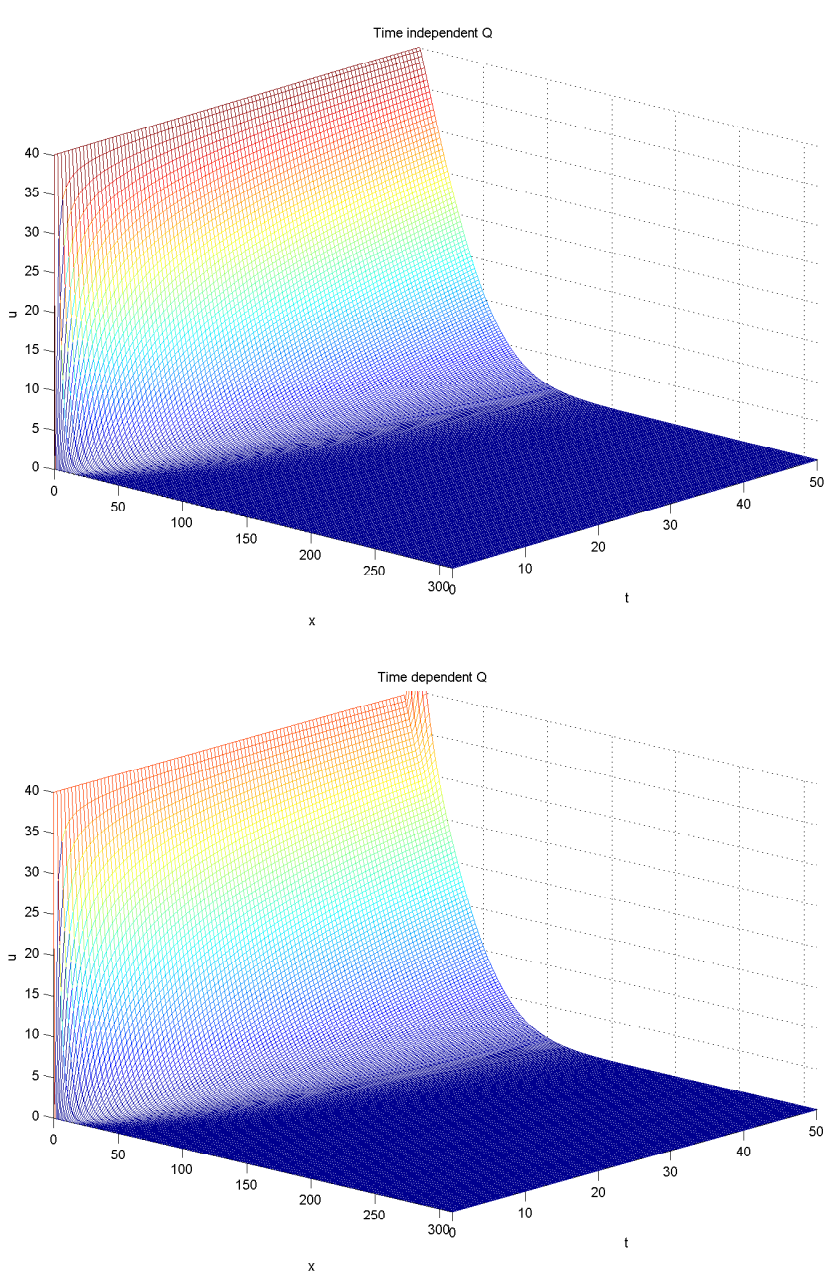

Figure 1: Numerical solution of equation 1 using the optimal control $Q$ for the time independent and time dependent case.

As we point out in the previous section, and according to our numerical results presented in [1], we may conclude that, for the time independent case, the injections must be done in the beginning of the chamber in order to minimize the given cost function. For the case where $Q$ is time dependent the computational results present similar behavior. However, the cost is reduced due to the fact that a great among of substance is injected late in time.

The solution $U=\left[U^{j}\right]_{j=0}^{m}$ of equation (7) for the optimal control $Q$ for both strategies are depicted in Figure 1. As we may see, the results are similar. However, for the time independent case, due to the fact that the injections are consider since the beginning, the solution exhibits a slower decreasing.

\section{Fitted solution}

The previous results allows to estimate $u$ in a discrete set of points, but they not allow to obtain an expression defined in $[0, L] \times[0, T]$. In this section, considering one injection point $\gamma \in[0, L]$, we obtain the best approximation of $u$ in a given class of continuous functions, depending on several real parameters. These parameters are obtained by the least-squares method applied to the mesh points. The solutions obtained are well adapted to the solution of equation (1). At first, we consider the case where $q$ is null function and we fit $u(x, t)$ by $v(x, t)=c_{0} e^{-p x / t}$, obtaining $p=1.034131$. The numerical and the fitted solutions are depicted in Figure 2. We proceed in the same way when we consider $q$ as a time independent/dependent function, considering the injection point 

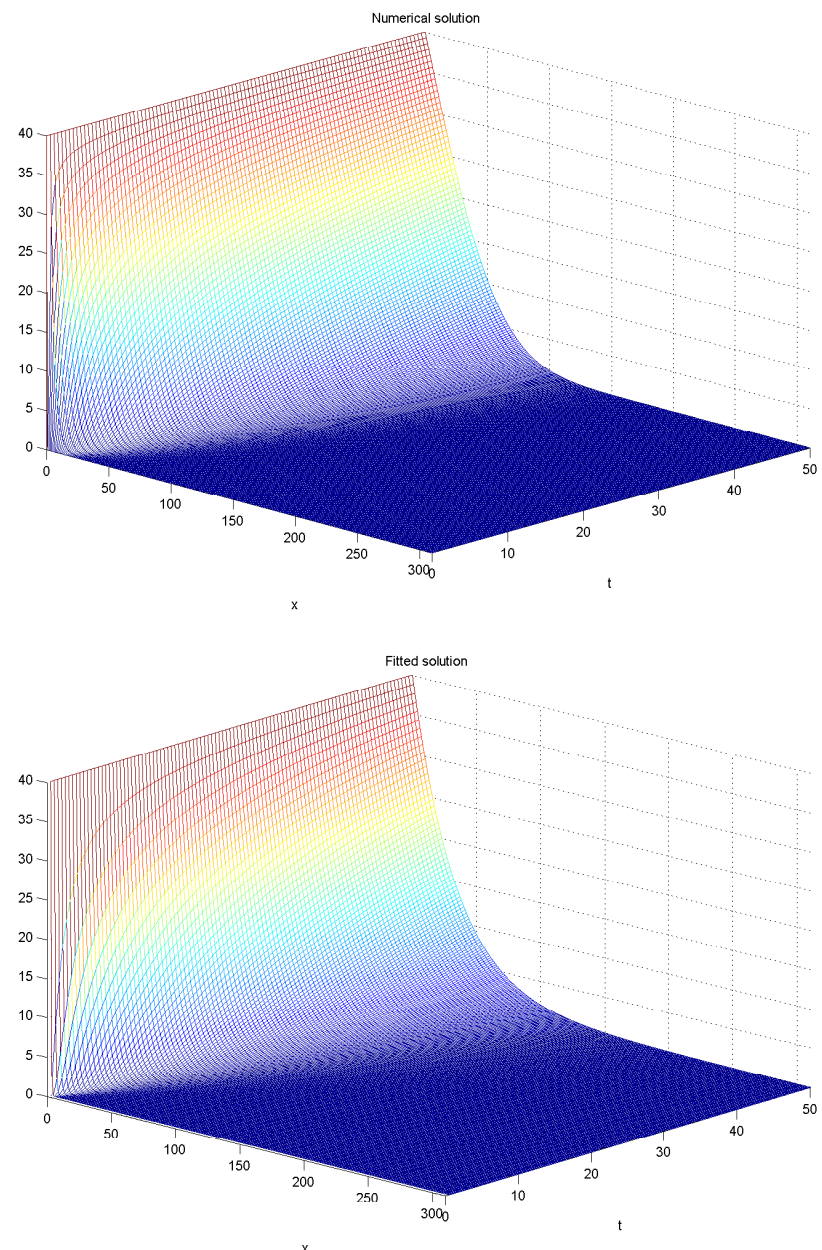

Figure 2: Numerical solution of equation (7) with out source font and the correspondent fitted solution $v(x, t)=c_{0} e^{-p x / t}$ with $p=1.034131$.

\begin{tabular}{c|ccc|} 
error & $Q=0$ & time indep. $Q$ & time dep. $Q$ \\
\hline$\|\mathcal{R}(E(v))\|$ & 0.01107 & 0.02377 & 0.02298 \\
$\| U-\mathcal{R}(v \|$ & 0.01547 & 0.01035 & 0.01064 \\
\hline
\end{tabular}

Table 1: Error for the fitted solution on the mesh points.

$\gamma=150$. The computational results are presented in Figures 3 and 4 and the errors are reported in Table 1, where

$$
E(v)=v_{t}-\alpha v_{x x}-q(t) \delta(x-\gamma),
$$

$\mathcal{R}$ is the restriction operator to the mesh points and $\|\cdot\|$ is the euclidian norm. According to the results, we may conclude that this technique is very efficient since we may obtain good continuous approximations to the exact solution of (1).

\section{Conclusions}

In this work, we study a diffusion equation with discrete source terms which may be interpreted as a model for the oxygen diffusion in a microbial fuel cell. We also consider an approximation of the numerical solution in a special class of continuous functions.

According to our numerical results, and the theoretical ones presented in [1], we conclude that, in order to minimize the given cost function, the injections must be done in the begin-
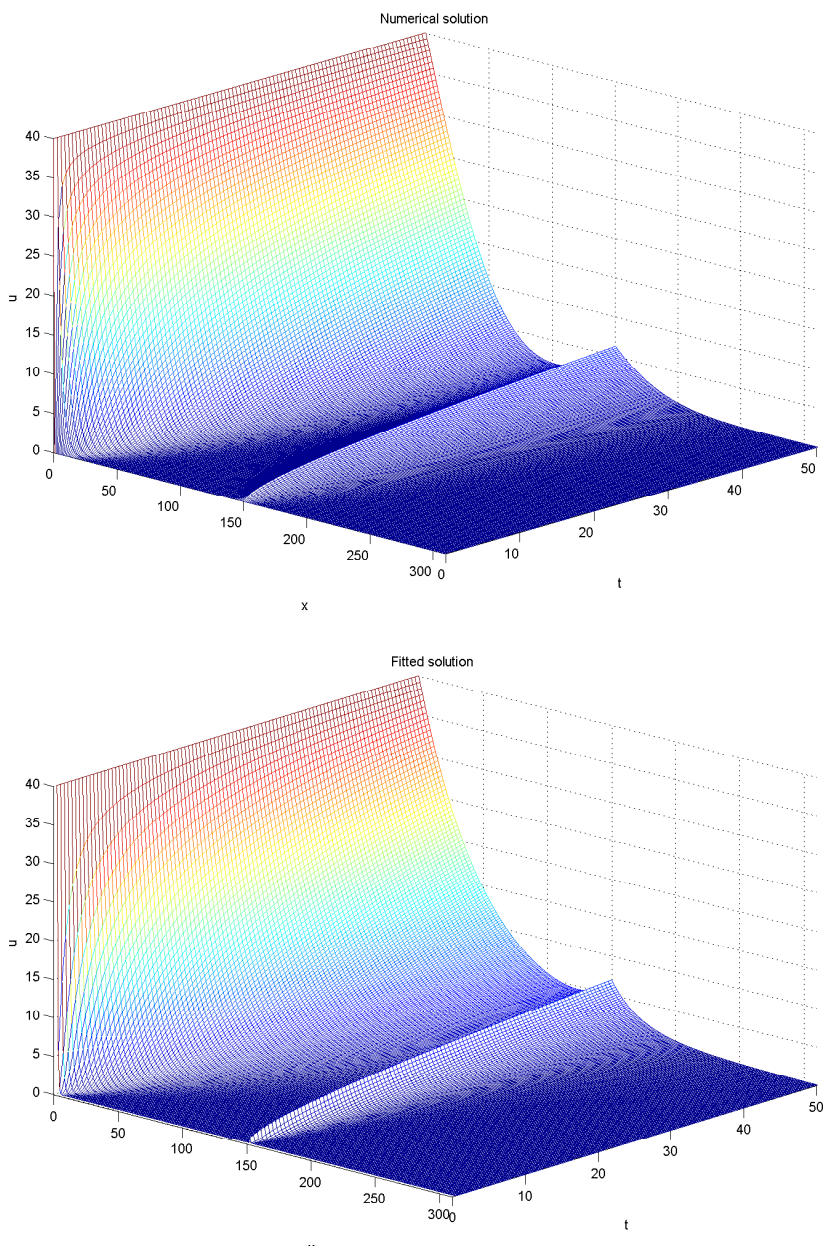

Figure 3: Numerical solution of equation (7) with one source font at position $\gamma=150$ and the correspondent fitted solution $v(x, t)=c_{0} e^{-p_{1} x / t}+\left(t^{p_{2}}-c_{0} e^{-p_{1} \gamma / t}\right) e^{-p_{3}|x-\gamma| / t}$ with $p_{1}=1.012630, p_{2}=0.494404$ and $p_{3}=2.533110$ (time independent case). 


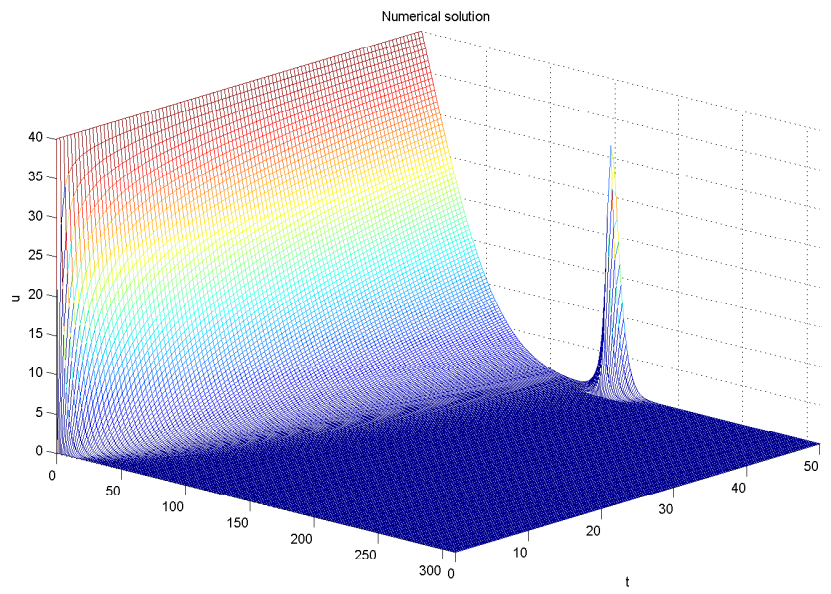

ning of the chamber, and near the final time (for the time dependent case).

The technique used to obtain a continuous approximation to the numerical/exact solution is very efficient and can be easily adapted to fit other functions.

\section{References}

[1] A. Araújo, Maria F. Patrício and José L. Santos. Optimal solution of a diffusion equation with a discrete source term. DMUC report $07-33$.

[2] R.P. Beyer and R.J. Leveque. Analysis of a onedimensional model for the immerse d boundary method. SIAM J. Numer. Anal., 29:332-364, 1992.

[3] Geun-Cheol Gil, In-Seop Chang, Byung Hong Kim, Mia Kim, Jae-Kyung Jang, Hyung Soo Park, and Hyung Joo Kim. Operational parameters affecting the performance of a mediator-less microbial fuel cell. Biosensors and Bioelectronics, 18:327-334, 2003.

[4] J.D. Kandilarov and L.G. Vilkov. The immersed interface method for a nonlinear chemical diffusion equation with local sites of reactions. Numerical Algorithms, 36:285307, 2004.

[5] S. Nash and A. Sofer. Linear and nonlinear programming. Mc. GrawHill, New York, 1996.

[6] J. Nocedal and S. Wright. Numerical Optimization. Springer, New York, 1999.

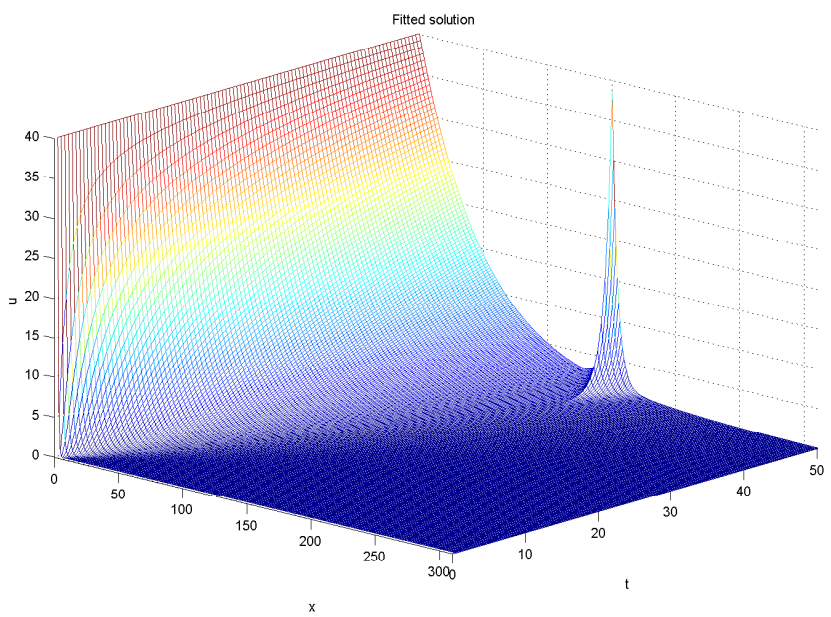

[7] Eric A. Zielke. Numerical Analysis of a one dimensional Diffusion Equation for a single chamber Microbial Fuel Cell using a Linked Simulation Optimization (LSO) technique. Advanced Numerical Methods, E521, 2006.

Figure 4: Numerical solution of equation (7) with one source font at position $\gamma=150$ and the correspondent fitted solution $v(x, t)=c_{0} e^{-p_{1} x / t}+p_{2} e^{t-T} e^{-p_{3}|x-\gamma| / t}$ with $p_{1}=1.033471$, $p_{2}=12.286661$ and $p_{3}=36.749810$ (time dependent case). 